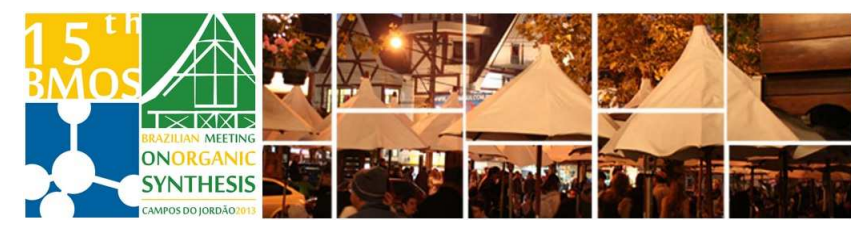

\title{
Synthesis and reactivity of pyrazolo-pyridone ring,
}

\section{an understudied heteroaromatic scaffold}

\author{
Paulo Eliandro da Silva Júnior ${ }^{1}$, A. Ganesan ${ }^{2}$, James Dale ${ }^{3}$, Flavio Emery ${ }^{1}$ \\ ${ }^{1}$ Faculty of Pharmaceutical Sciences of Ribeirao Preto, University of Sao Paulo, Brazil; ${ }^{2}$ School of \\ Pharmacy, University of East Anglia, UK; ${ }^{3}$ NIBR, GDC Horsham, UK \\ *paulo.silva@fcfrp.usp.br
}

Keywords: pyrazolo-pyridone, $\mathrm{N}$-acyl-N-nitroso rearrangement, heterocycle

\section{INTRODUCTION}

The chemical structural space of all possible compounds is vast, with more than $10^{62}$ compounds $^{1}$ with molecular weights below 500 Da. Heteroaromatic rings are common in bioactive molecules and both medicinal and agro chemists have exploited these scaffolds to provide high value materials, for example Glivec, an anti cancer drug, and Exjade to treat iron overload. However, only a small fraction of the heteroaromatic space is currently exploited for drug discovery, and has established synthetic routes to access them, supporting a scenario in which is shown the disclosure of new heterocyclic skeletons follows a rate of 5-10 per year. The main scope of this work is to study the synthesis of underexplored heterocyclic scaffold, based on a recent computational study ${ }^{2}$, which listed interesting yet under represented heterocyclic skeletons with drug discovery potential.

\section{RESULTS AND DISCUSSION}

The selected scaffold is a pyrazolo-pyridone 1 , it is a 6,5 fused ring system with mixed electronic characteristics. The synthesis of the target heterocycles had been achieved by an N-acyl-Nnitroso rearrangement approach (Scheme 1$){ }^{3}$

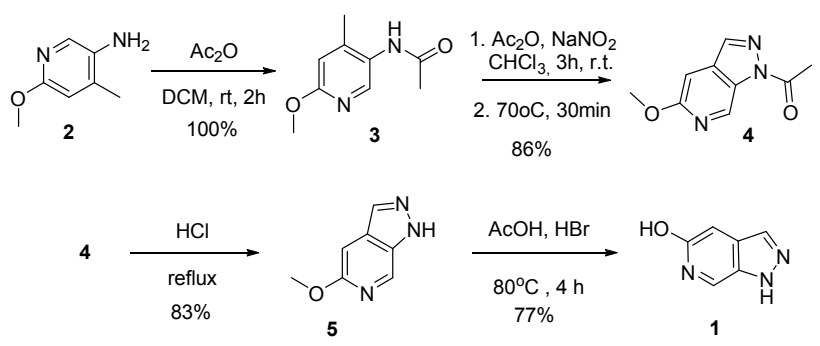

Scheme 1. Synthesis of the pyrazolo-pyridinone ring system through the cyclisation of a $\mathrm{N}$-acyl-N-nitroso intermediate.

Once a suitable synthetic methodologies have been found, the core's reactivity had been studied under a range of synthetically useful transformations (Scheme 2).

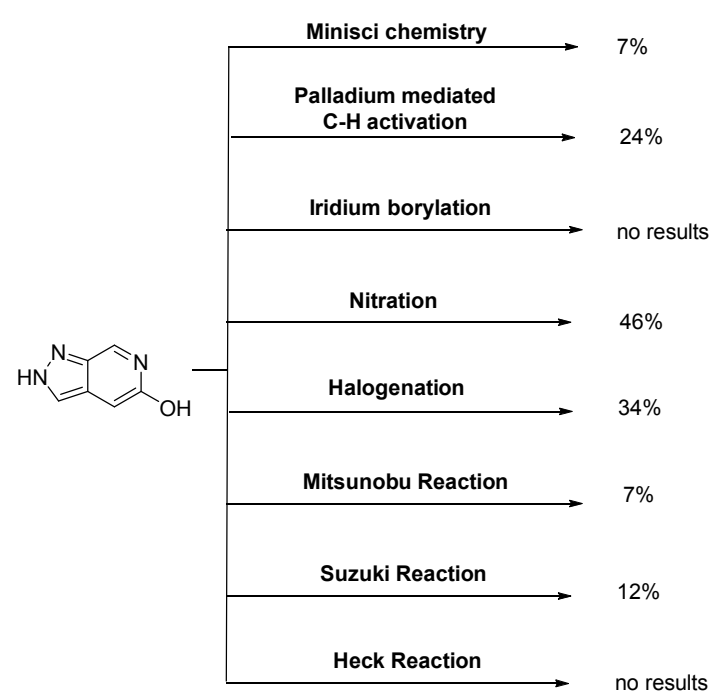

Scheme 2. Exploratory studies about the reactivity of pyrazolo-pyridine rings.

\section{CONCLUSION}

The selected heteroaromatic ring represents a promising scaffold to obtain libraries, based on its reaction capacity in several positions, revealing its versatility. However, further studies are needed to improve conditions and yields.

\section{ACKNOWLEDGEMENTS}

I want to acknowledge and thank the CAPEs for the scholarship, besides FAPESP, CNPq, the Novartis D\&I Office.

\section{REFERENCES}

${ }^{1}$ Leeson, P.D.; Davis, A.M.; Steele, J. Drug Discovery Today: Technol. 2004, 1, 189-195.

${ }^{2}$ Pitt,W.R.; Parry, D.M.; Perry, B.G.; Gnonm, C.R.. J. Med. Chem. 2009 , 52, 2952-2963.

3 Tsikouris, O. et al. Magn. Reson. Chem. 2008, 46, 643-649. 